\title{
Risk Assessment due to Electricity Price Forecast Uncertainty in UK Electricity Market
}

\author{
Gao Gao, Kwoklun Lo and Jianfeng Lu \\ Department of Electronic and Electrical Engineering \\ University of Strathclyde \\ Glasgow, United Kingdom \\ gao.gao@strath.ac.uk
}

\begin{abstract}
This paper illustrates the risk assessment on electricity price forecast uncertainty. The high-risk periods under different time have been indicated. Autoregressive integrated moving average (ARIMA) models and artificial neural network (ANN) techniques are introduced to forecast electricity prices in UK electricity market. Also, this paper investigates the risk index of electricity prices due to forecast uncertainties in the competitive power market through two aspects - daily and seasonal. This risk index is calculated using the errors of short-term electricity price forecast. The input data of forecasting models is divided into weekday and weekend profiles and this is done to observe the different electricity price dynamic risks between weekdays and weekends.
\end{abstract}

Index Terms--Electricity market, Electricity price forecasting, Risk assessment, Risk index.

\section{INTRODUCTION}

In the late 1980s, UK took the lead in implementing industry privatization reform and proposed deregulation of the electricity power supply industry. From 1990 to 2001, the National Grid Company (NGC) was founded, and arrangements called the Electricity Pool operated for the production, purchasing and trading of wholesale electricity. On 27 March 2001, the New Electricity Trading Arrangements (NETA) were put in place, but it only represented the wholesale electricity market for England and Wales [1]. After 2005, the British Electricity Trading and Transmission Arrangements (BETTA) replaced NETA and covered the whole area of the UK [2].

There are two forms of transactions between generators and operators in UK power wholesale market - bilateral contract and through a Power Exchange (PE). For bilateral contract, trade parties sign a bilateral contract by negotiation, and determine the prices and volumes of the transaction. Power Exchange is a centralized power-trade place. Generators and operators submit their offers that contain prices and capacities of energy to be traded. Then PE integrates and clears these offers. In the UK, $77 \%$ of the electricity in the wholesale market is traded bilaterally. The other $23 \%$ are implemented on exchange. Power Exchange consists of three sub-markets, day-ahead auction, spot market and prompt market. And most of the traded electricity is accomplished by day-ahead auction [3].
Therefore electricity price forecasting has become important to promote competition and to guarantee the benefit of participants in the market [4]. As market participants, both generators and operators intent to contribute more efforts on developing appropriate price forecasting scheme to maximize their profits. If the electricity price can be forecasted accurately, generation side could handle the market dynamically and make an optimal strategy of power generation. In the meanwhile, the demand side could consume the electricity energy within a particular time slot when lower electricity price is forecasted. It therefore could reduce the total cost and improve the market competitiveness [5].

In the past many forecasting methods and models were used in different applications. These methods were generally divided into two categories: logical judgment and mathematical model. Also mathematical models are classified into causal model and time series model [6].

In this paper, Autoregressive integrated moving average (ARIMA) models and artificial neural network (ANN) models are used to forecast electricity price. And the hourly updated electricity prices from UK day-ahead auction market are regarded as the experimental data, which are obtained from UK N2EX, Nord Pool [7]. The one-year auction prices from March 2015 to February 2016 are used as the experimental data here. First of all, the historical electricity prices are classified into two parts, weekday and weekend profiles. This is to observe the different electricity price characteristics between weekdays and weekends. Then the one-year historical prices are classified into 12 months, and the forecast processes are performed on each month. For each month, the prices in the first three weeks are used for training, and the data in the last week are used for testing.

In addition to forecasting models, another significant concern is to investigate the risk index of trade parties due to the errors from electricity price forecasts. Risk assessment is becoming increasingly important in power market because it could help the market participants to evaluate their financial risk under uncertainty [8]. The electricity prices are updated hourly, so there are 24 auction prices every day. Also there are 24 forecast prices per day. Then the risks can be classified into 24 periods a day. It could be used to observe the daily 
risk distribution over a period and can be weekdays or weekends. And the seasonally risk distributions are also performed.

\section{Methodology}

\section{A. Autoregressive Integrated Moving Average Model}

The traditional ARIMA model was first studied in the 1920s. When George Box and Gwilym Jenkins published their research results in 1970, the research and application of time series method has stepped into a new stage [9].

ARIMA model is made up of the integrate process and ARMA model. For an ARIMA (p,d,q) model, the orders $p$ and $q$ represent the numbers of autoregressive terms and moving average terms separately and $d$ is the level of differencing which ensures the stationarity of the time series. The basic ARIMA model can be presented by the following expression:

$$
\emptyset(B)(1-B)^{d} z_{t}=\theta_{0}+\theta(B) a_{t}
$$

where $\emptyset(B)$ is the operator of $p$ and $\theta(B)$ is the operator of q. $B$ is the lag operator, $z_{t}$ is the historical auction electricity price at time $t$ and $\theta_{0}$ is the constant term. $a_{t}$ is the error term which is generally assumed to be independent and its average value is zero.

If the historical data indicates non-stationarity, a differencing step is necessary to be used to convert the data to a stationary time series, which is the integrated part of the model. The differencing step can be applied more than once until the data presents stationarity. The first order and $\mathrm{d}^{\text {th }}$ order difference can be expressed as:

$$
\begin{gathered}
\nabla z_{t}=z_{t}-z_{t-1} \\
\nabla^{d} z_{t}=\nabla^{d-1} z_{t}-\nabla^{d-1} z_{t-1}
\end{gathered}
$$

where $\nabla z_{t}$ and $\nabla^{d} z_{t}$ are the difference equations for first order and $\mathrm{d}^{\text {th }}$ order respectively. In most cases, when the value of $d$ is 1 or 2 , the differenced time series could become stationary [10].

In the ARIMA model, autocorrelation function (ACF) and partial autocorrelation function (PACF) are used to check the order of $p$ and $q$. The moving average order $q$ is determined by ACF, and the autoregressive order $p$ is decided by PACF. For the stationary time series, ACF will decay rapidly to zero with increasing the number of delays. According to the observations of ACF and PACF, different values of orders $p$ and $q$ can be selected to create several ARIMA models [11]. Then the optimal model with the best result from these models is used to make the forecasting.

\section{B. Artificial Neural Network model}

Artificial neural network (ANN) is the research hotspot in the field of artificial intelligence since the 1980s. It can simulate the interaction of biological nervous systems to realworld objects. With the deepening of ANN research work, ANN techniques have been used widely in many different areas, such as intelligent robot, pattern recognition, automatic control and forecast estimation [12].

In general, the most widely used structure of ANN model is the multilayer feed-forward network, which includes input layer, hidden layer and output layer respectively[13]. A threelayer feed forward neural network is illustrated in Figure 1. In forecast applications, the original data is usually classified into training part and testing part. The training part is used for constructing the neural network, and the testing part can test the trained model.

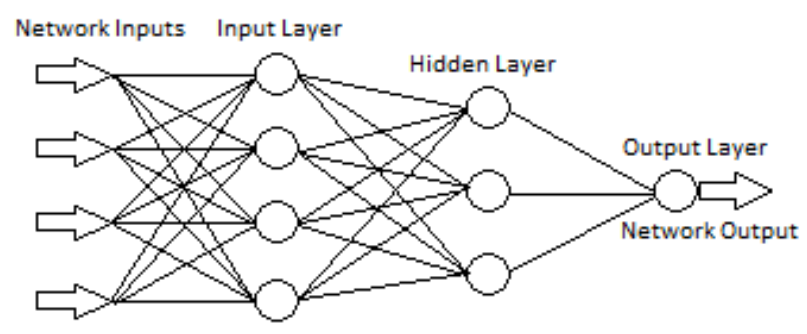

Figure 1. Three-layer artificial neural network architecture.

Neural network is an arithmetic model, and composes of a large number of neurons. And each neuron represents a specific output function, which is also known as an activation function. The connection between two neurons indicates a weighted value for the signal that is passing through them, and this is equivalent to the memory of ANN. Every neuron in the network sums its weighted inputs to produce an internal activity level $v_{i}$ :

$$
v_{i}=\sum_{j=1}^{n} w_{i j} x_{i j}-w_{i 0}
$$

where $w_{i j}$ is the weight of the connection from input $j$ to neuron $i, x_{i j}$ is the input signal number from $j$ to $i$, and $w_{i 0}$ is the threshold associated with unit $i$. The output of neuron $y_{i}$ is expressed as

$$
y_{i}=\varphi\left(v_{i}\right)
$$

where $\varphi\left(v_{i}\right)$ is the defined function expression. It has many different forms in different situations [14]. During the training process, the network learns through adjusting both the weights connecting the input and hidden layer and the weights connecting the hidden layer and the output, by the gradient multiplied by the learning rate parameter. In the application in this paper the best forecasting results of ANN models are selected after training more than 100 times respectively.

\section{NUMERICAL FORECASTING RESULTS}

In this work, the historical auction prices from March 2015 to February 2016 in UK have been used. As discussed previously, the electricity price forecasting model is categorized as weekdays and weekends respectively (2 parts). Moreover, ARIMA and ANN models (2 models) are all used to forecast both weekday and weekend data in each month and season. Therefore, at least 48 models ( 2 models/part $\times 2$ 
parts/month $\times 12$ months) are required to obtain the best forecast results over one year. The one-hour-ahead electricity price forecasting has been implemented in this paper.

In order to compare the forecasting accuracy of each forecasting model, the mean absolute percentage error (MAPE) and the root mean square error (RMSE) are applied to assess the errors between forecast values $f_{t}$ and actual values $y_{t}$. If $n$ is the number of hours. Their functions are expressed as

$$
\begin{gathered}
M A P E=\frac{1}{n} \sum_{t=1}^{n}\left|f_{t}-y_{t}\right| / y_{t} \\
R M S E=\sqrt{\frac{\sum_{t=1}^{n}\left(f_{t}-y_{t}\right)^{2}}{n}}
\end{gathered}
$$

Here, the forecast results from ARIMA and ANN models on September 2015 are used as an example. The historical auction price data on weekdays from 1 to 18 September 2015 are used as training data to forecast the electricity prices from 21 to 25 September 2015. And the historical data on weekends from 5 to 20 September 2015 are used as training data to forecast the electricity prices from 26 to 27 September 2015. And the results of forecast errors are presented in Table I.

TABLE I

COMPARISON BETWEEN THE FORECAST ERRORS FOR WEEKDAYS AND WEEKENDS

\begin{tabular}{|c|c|c|c|c|}
\hline \multirow{2}{*}{ Time Period } & \multicolumn{2}{|c|}{ MAPE for weekdays } & \multicolumn{2}{c|}{ MAPE for weekends } \\
\cline { 2 - 5 } & ARIMA, $\%$ & $\boldsymbol{A N N}, \boldsymbol{\%}$ & ARIMA, $\%$ & ANN, $\%$ \\
\hline 00:00-01:00 & 14.75 & 16.21 & 5.66 & 12.82 \\
\hline 01:00-02:00 & 10.97 & 13.64 & 1.46 & 20.75 \\
\hline 02:00-03:00 & 14.74 & 14.07 & 8.71 & 3.00 \\
\hline 03:00-04:00 & 6.49 & 3.46 & 5.50 & 3.15 \\
\hline 04:00-05:00 & 8.66 & 2.05 & 1.98 & 3.20 \\
\hline 05:00-06:00 & 8.45 & 8.05 & 2.26 & 0.97 \\
\hline 06:00-07:00 & 8.96 & 3.29 & 2.16 & 2.06 \\
\hline 07:00-08:00 & 5.61 & 3.07 & 3.86 & 1.51 \\
\hline 08:00-09:00 & 5.67 & 6.64 & 3.05 & 1.87 \\
\hline 09:00-10:00 & 2.97 & 6.18 & 0.94 & 8.25 \\
\hline 10:00-11:00 & 11.27 & 7.23 & 16.73 & 11.40 \\
\hline 11:00-12:00 & 6.55 & 4.78 & 13.59 & 12.73 \\
\hline 12:00-13:00 & 13.65 & 10.58 & 10.20 & 3.65 \\
\hline 13:00-14:00 & 4.54 & 6.16 & 8.24 & 13.73 \\
\hline 14:00-15:00 & 7.52 & 4.79 & 13.34 & 19.24 \\
\hline 15:00-16:00 & 7.58 & 7.12 & 5.06 & 7.17 \\
\hline 16:00-17:00 & 8.81 & 6.51 & 6.89 & 3.75 \\
\hline 17:00-18:00 & 7.44 & 3.67 & 2.00 & 3.48 \\
\hline 18:00-19:00 & 9.68 & 11.63 & 8.85 & 7.48 \\
\hline 19:00-20:00 & 11.07 & 8.17 & 8.31 & 3.01 \\
\hline 20:00-21:00 & 18.06 & 13.29 & 19.46 & 14.84 \\
\hline 21:00-22:00 & 29.96 & 29.42 & 35.37 & 27.76 \\
\hline 22:00-23:00 & 28.06 & 10.71 & 70.89 & 61.86 \\
\hline 23:00-24:00 & 10.74 & 10.55 & 18.77 & 13.43 \\
\hline Average & 10.92 & 9.22 & 11.39 & 10.88 \\
\hline RMSE(£/MWh) & 7.5180 & 6.8776 & 10.6796 & 9.7712 \\
\hline & & & & \\
\hline & & & \\
\hline
\end{tabular}

The electricity price forecasts for weekdays and weekends by ARIMA and ANN are shown in Figure 2, 3, 4 and 5 respectively.

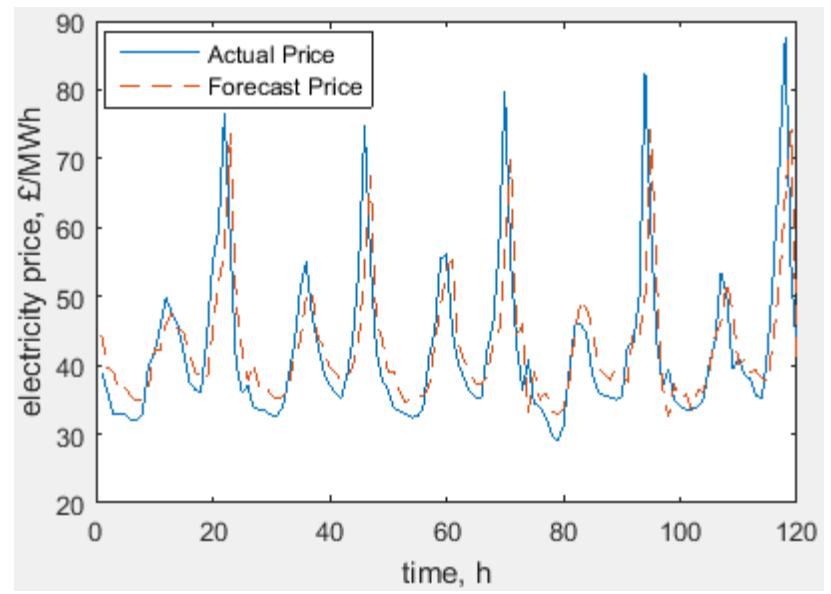

Figure 2. Electricity price forecasts for 21-25 September 2015 on weekdays by ARIMA model.

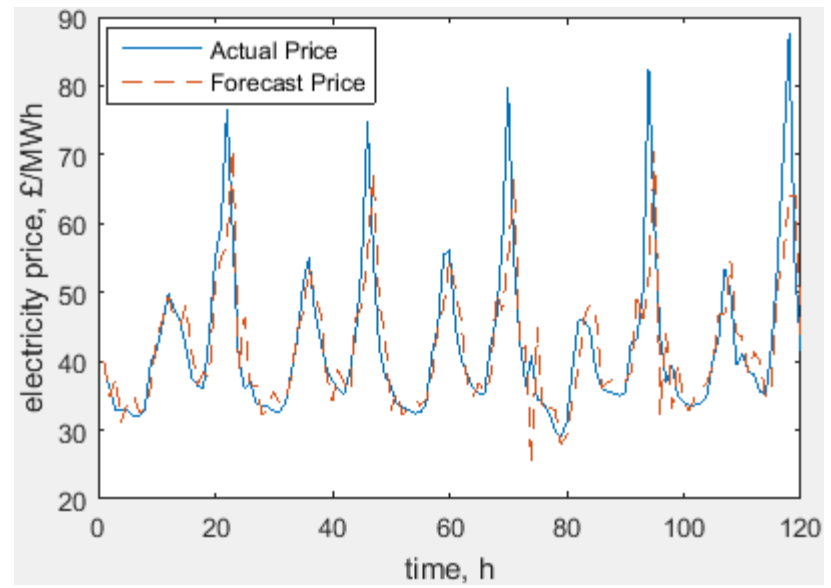

Figure 3. Electricity price forecasts for 21-25 September 2015 on weekdays by ANN model.

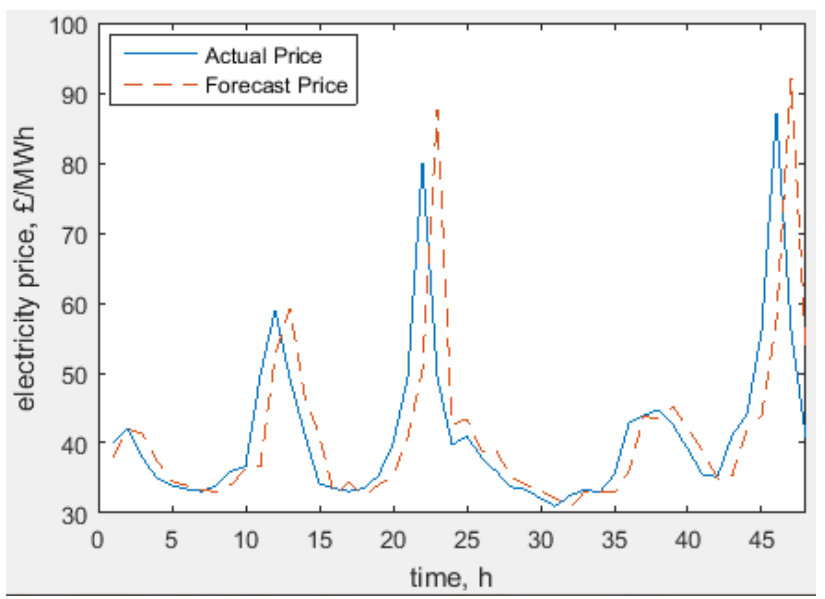

Figure 4. Electricity price forecasts for 26-27 September 2015 on weekends by ARIMA model.

It is observed from Table I that the MAPE for weekdays stays in a range of 9.22-10.92\% and the MAPE for weekends is $10.88-11.39 \%$. Furthermore, the RMSE of weekdays is from 6.8776-7.5180 $£ / \mathrm{MWh}$ and for weekends this increases 


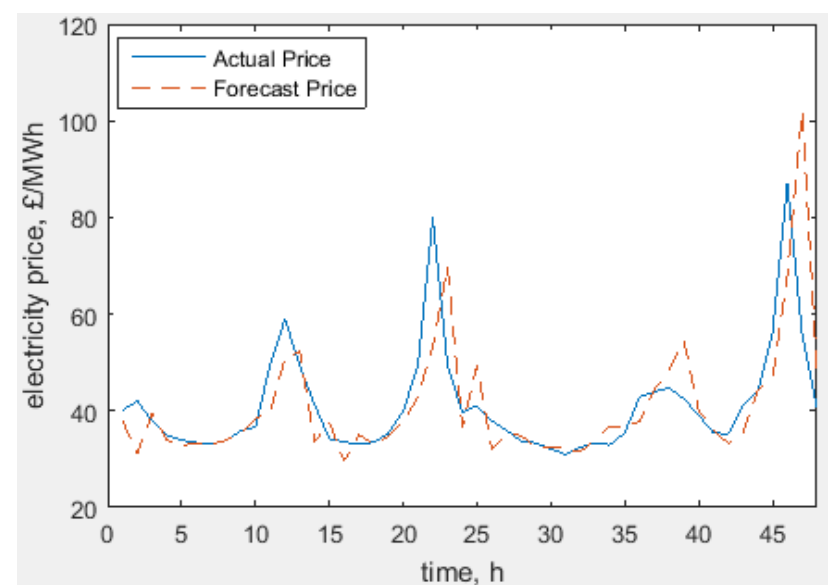

Figure 5. Electricity price forecasts for 26-27 September 2015 on weekends by ANN model.

to $9.7712-10.6796 £ / \mathrm{MWh}$ respectively. The results indicated that the forecast for weekends are more difficult than weekdays. This is because electricity prices fluctuate more on weekends. And the RMSEs and average MAPEs of ANN models are all smaller than ARIMA models. That means on the forecasting of September 2015, ANN models performed better than ARIMA models.

In addition, as can be observed from Figure 2, 3, 4, 5 and Table I, at most of the times, the forecasting errors of both ARIMA and ANN models are similar. In order to prove this, the result of weekdays' and weekends' daily average forecasting errors in September 2015 that are achieved by ARIMA and ANN model respectively are shown in Figure 6 and Figure 7. The Figures illustrate that the error lines produced by ARIMA and ANN models have very similar trends.

Also, we can found that some bigger forecasting errors in some specific periods during a whole day no matter which model is used. For weekdays, the peak forecast error appears at period 22, and for weekdays, 22nd and 23rd period are shown bigger errors than other periods with both forecasting models.

Based on these periods with high forecast errors, the highrisk peaks can be observed during 24 periods in a day. And the risk index could be analysed due to the electricity price forecast errors. All the forecast and comparison processes in this section are used on every month from March 2015 to February 2016.

\section{RISK ANALYSIS DUE TO ELECTRICITY PRICES FORECASTING ERRORS}

Although it has strong correlations between the forecast errors by ARIMA and ANN models, there must be a model that forecast more accurately. Table II shows the average MAPE comparison between ARIMA and ANN models. It can be observed form this Table that almost all the average MAPEs of ANN are smaller than ARIMA, no matter they are for weekdays or weekends. It means that ANN models have

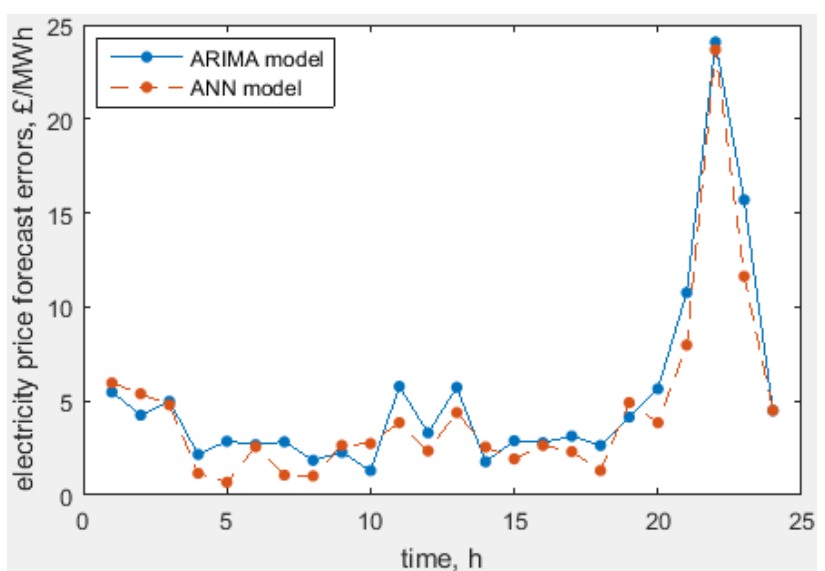

Figure 6. Comparisons of weekdays' daily electricity price forecast errors by ARIMA and ANN for September 2015.

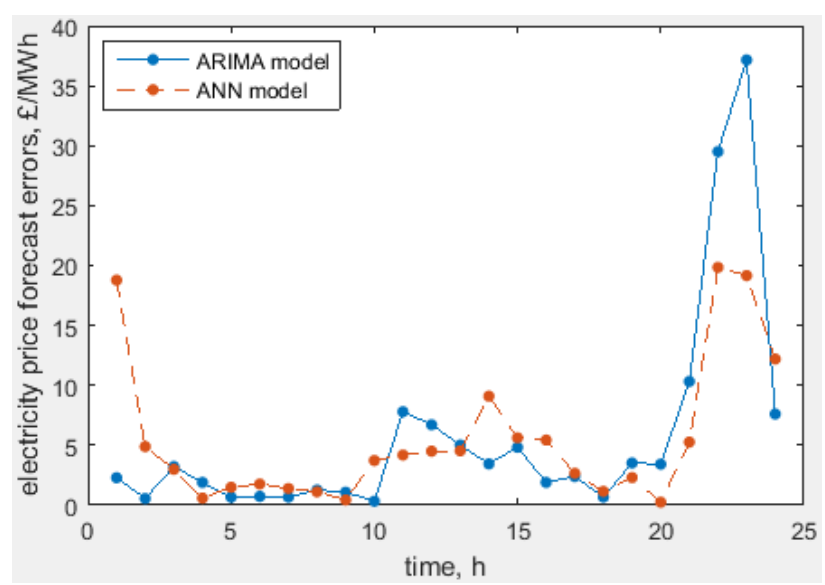

Figure 7. Comparisons of weekends' daily electricity price forecast errors by ARIMA and ANN for September 2015.

TABLE II

AVERAGE MAPE COMPARISON BETWEEN ARIMA AND ANN MODELS FOR WEEKDAYS AND WEEKENDS

\begin{tabular}{|c|c|c|c|c|}
\hline \multirow{2}{*}{ Month } & \multicolumn{2}{|c|}{ Avg. MAPE for weekdays } & \multicolumn{2}{c|}{ Avg. MAPE for weekends } \\
\cline { 2 - 5 } & $\boldsymbol{A R I M A , \%}$ & $\boldsymbol{A N N}, \boldsymbol{\%}$ & $\boldsymbol{A R I M A , \%}$ & $\boldsymbol{A N \boldsymbol { N } , \boldsymbol { \% }}$ \\
\hline 2015.03 & 11.60 & 8.77 & 18.22 & 16.51 \\
\hline 2015.04 & 9.25 & 8.28 & 10.98 & 10.34 \\
\hline 2015.05 & 9.45 & 7.94 & 13.48 & 21.19 \\
\hline 2015.06 & 7.82 & 6.92 & 10.75 & 10.18 \\
\hline 2015.07 & 7.88 & 6.94 & 13.08 & 12.66 \\
\hline 2015.08 & 8.62 & 8.51 & 10.21 & 9.58 \\
\hline 2015.09 & 10.92 & 9.22 & 11.39 & 10.88 \\
\hline 2015.10 & 11.06 & 13.26 & 17.83 & 13.49 \\
\hline 2015.11 & 13.05 & 12.90 & 21.40 & 20.55 \\
\hline 2015.12 & 16.31 & 15.29 & 33.55 & 27.80 \\
\hline 2016.01 & 18.03 & 13.45 & 18.89 & 22.19 \\
\hline 2016.02 & 16.19 & 14.87 & 14.54 & 19.51 \\
\hline
\end{tabular}

better forecasting accuracy than ARIMA models in this set of data. Therefore it will be focused on the forecasts using ANN models to make the risk analysis in this section.

From the forecast results we can see the errors of electricity price forecast play an important role in the risk assessment 
due to forecasting uncertainty [15]. For risk assessment, all the forecast errors are represented by risk indexes. The range of risk index is from 0 to 1 . It is expressed as the ratio of the errors in each period to the maximum forecast errors during a day.

In this paper, there are 24 risk indices as the data are hourly updated. The risk indexes have been accomplished for every month respectively during March 2015 to February 2016. And the average daily errors of the year have been calculated. Then the 24-period risk index of weekdays and weekends for the whole year can be achieved, which is shown in Figure 8 and Figure 9 respectively. Additionally, the risk indexes are illustrated seasonally to observe the influence due to different seasons. These are indicated in Figure 10 and Figure 11 respectively.

It is illustrated in Figure 8 and Figure 9 that there are more irregular risk peaks appeared on weekends and its average risk value is higher than the values at weekdays. These bigger uncertainties indicate that the forecasts on weekends are more difficult than weekdays. But during the time periods from 20 to 23 , both figures indicate high risks at those periods and their value peaked at at 21:00 and 20:00 respectively. It means the high electricity price forecast risks are more likely to happen from 20:00 to 23:00 in a day, no matter the forecasting is for weekdays or weekends.

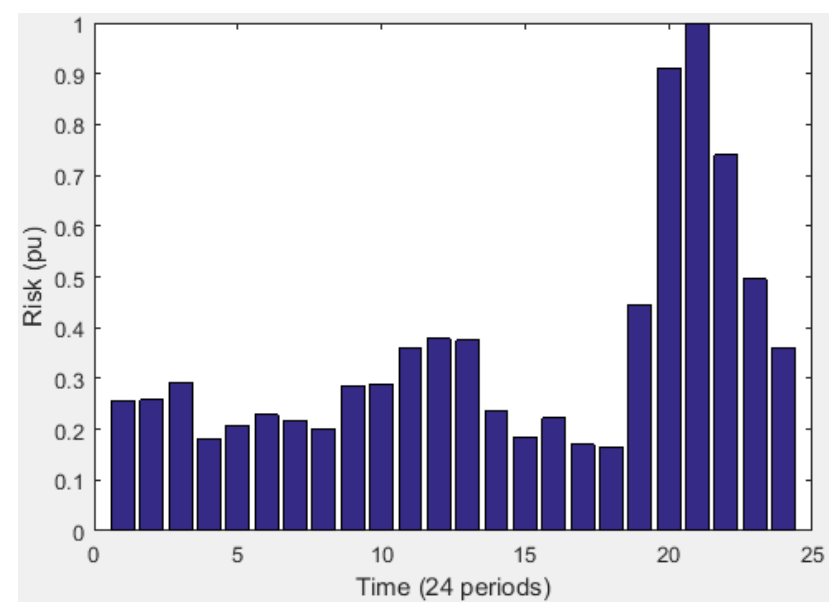

Figure 8. Risk index of electricity price forecast errors on weekdays.

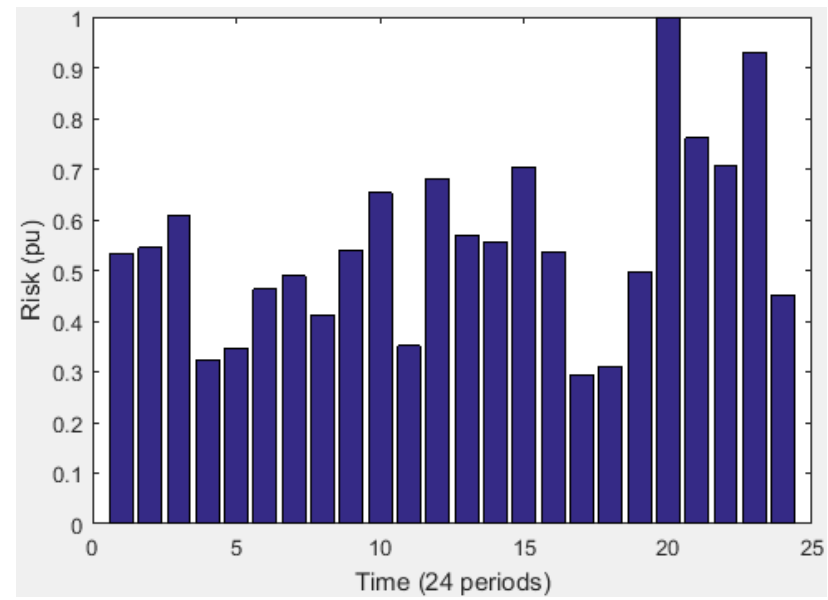

Figure 9. Risk index of electricity price forecast errors on weekends.

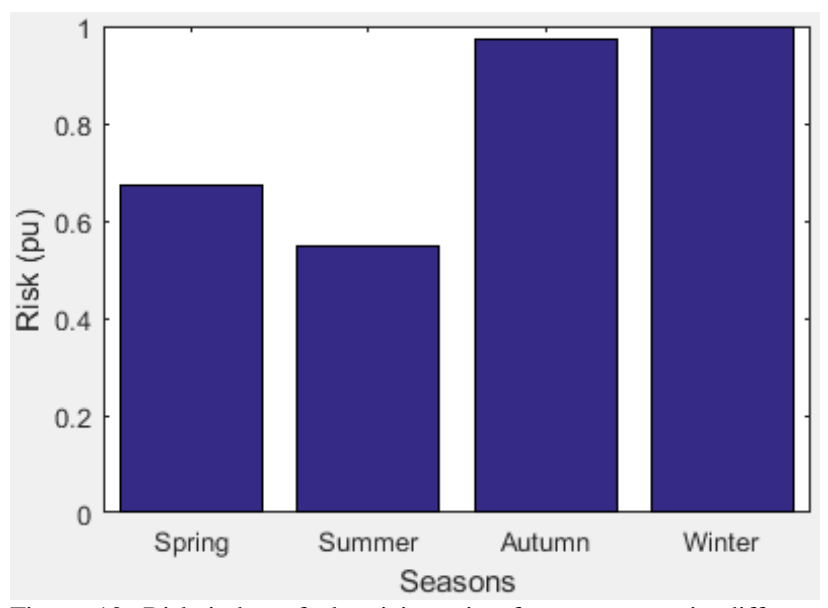

Figure 10. Risk index of electricity price forecast errors in different seasons on weekdays.

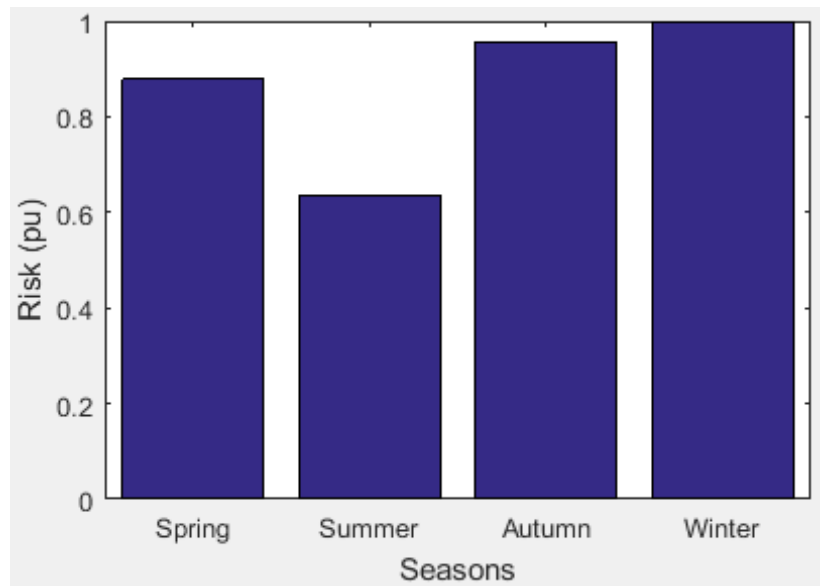

Figure 11. Risk index of electricity price forecast errors in different seasons on weekends.

In Figure 10 and 11, the risk indexes are shown in different seasons. For weekdays, the risk index in winter is higher than the other seasons. And the risk index in autumn is also very high, it is only a little smaller than in winter. For weekends, the peak value also occurs in winter and is followed by autumn. The risk index in spring and summer is smaller than that in autumn and winter for both forecasting. The average value of risk index on weekends is still bigger than weekdays.

\section{CONCLUSIONS}

This paper introduced and assessed the ARIMA and ANN forecasting models for electricity price forecast based on the day-ahead auction prices in UK electricity market. The forecasting process was divided into weekday and weekend parts. According to the forecasting accuracy in terms of MAPE and RMSE, it is observed that the electricity price forecast for weekends was more difficult than the forecast for weekdays owing to the uncertainty of weekends' electricity prices. Although the forecasting errors obtained by ARIMA and ANN models were similar in each month, ANN models shown better forecasting results in this paper. Finally, the risk 
indexes due to the electricity price forecasting errors over different time periods and seasons were presented. The overall daily and seasonally risk indexes on weekends performed bigger than weekdays.

\section{ACKNOWLEDGEMENTS}

The authors would like to thank UK N2EX, Nord Pool for supplying the historical day-ahead auction prices.

\section{REFERENCES}

[1] Monica Giuliettia, Luigi Grossib, Michael Waterson, "Price transmission in the UK electricity market: Was NETA beneficial?" Energy Economics, Volume 32, Issue 5, Pages 1165-1174, January 2010.

[2] Youngho Chang, Jian Liang Lee, "Electricity market deregulation and energy security: a study of the UK and Singapore electricity markets,' International Journal of Global Energy Issues, Volume 29, 2008.

[3] http://www.nordpoolspot.com/TAS/Rulebook-for-the-PhysicalMarkets/n2ex/

[4] M.Shafie-khah, M. Parsa Moghaddam, M.K. Sheikh-El-Eslami. "Price Forecasting of Day-ahead Electricity Markets Using a Hybrid Forecast Method," Energy Conversion and Management, 52, 2165-2169, 2011.

[5] Gao Gao, Jianfeng Lu, Fulin Fan and Kwoklun Lo. "A short-term Electricity Price Forecasting Scheme for Power Market." World Journal of Engineering and Technology, 4, 58-65, Oct. 2016.
[6] Yao Dong, Jianzhou Wang, He Jiang and Jie Wu. "Short-term electricity price forecast based on the improved hybrid model," Energy Coversion and Management, vol. 52, pp 2987-2995, Aug. 2011.

[7] http://www.nordpoolspot.com/historical-market-data/

[8] Dahlgren, R.W., Liu, C.C., and Lawarree, J. "Volatility in the California power market: source, methodology and recommendations," IEE Proc., Gener. Transm. Distrib., 148, (2), pp 189-193, 2001.

[9] J. Scott Armstrong, Forecasting by Extrapolation: Conclusions from Twenty-five Years of Research, vol. 14, Interfaces, Nov. 1984, pp 5266.

[10] G.E.P. Box, G.M. Jenkins, and G.C. Reinsel. Time Series Analysis: Forecasting and Control. 4th Ed, Oxfored: wiley.

[11] J. Contreras, R. Espinola, F. Nogales and .J. Conejo. "ARIMA Models to Predict Next-Day Electricity Prices," IEEE Transactions On power Systems, vol. 14, No.3, 2003.

[12] H.Y. Yamin, S.M. Shahidehpour, and Z. Li. "Adaptive short-term electricity price forecasting using artificial neural networks in the restructured power markets," International Journal of Electrical Power \& Energy Systems, vol. 26, pp 571-581, Oct. 2004.

[13] Hsiao-Tien Pao. "Forecasting electricity market pricing using artificial neural networks," Energy Conversion and Management, vol. 48, pp907-912, Mar. 2007.

[14] Ling Chen, Xu Lai. "Comparison Between ARIMA and ANN models Used in Short-term Wind Speed Forecasting," Power and Energy Engineering Conference (APPEEC), Asia-Pacific, Apr. 2011.

[15] K.L. Lo and Y. K. Wu. "Risk assessment due to local demand forecast uncertainty in the competitive supply industry," IEE Proc., Gener. Transm, Distrib., vol. 150, No. 5, Sep. 2003. 